\title{
Heat Dissipation Analysis of Bathtub based on Thermodynamics Binbin XIN
}

\author{
School of North China Electric Power University, Baoding071000, China; \\ 1766356865@qq.com
}

Keywords: Differential, Heat conduction, thermal radiation Thermal convection, Open system energy equation

\begin{abstract}
The physical model of the bathtub is established in this paper, and by using the differential theory of the water in the bathtub is regarded as the superposition of different temperature of the water layer, secondly, analysis of water bath heat dissipation form mainly has three kinds: the heat conduction through the wall heat loss, the bath room through the air and wall surface radiation heat loss and convection heat loss on the surface of the water. Finally, by using the energy equation of the open system in thermodynamics, the equation of the relationship between the heat and the heat loss when the water enters the bathtub is established. Finally, according to other conditions, we can get the amount of water needed for a bath.
\end{abstract}

\section{Introduction}

Bathtub is a kind of pipe device. People use it to take shower. It's usually located in the bathroom. Bathtub provides a comfortable experience However, the temperature of the bathwater will be decrease as time goes by. So it's necessary to add hot water steadily to remain the temperature. From the perspective of environmental protection, Whether saving water is one of the evaluation criteria of bathtub quality. The material, shape, and size are all the important factors to decide whether the bathtub save water or not. This question demand to develop a model of the temperature of the bathtub water in space and time, Try to keep the initial water temperature and find a best strategy of saving water.

\section{The Model}

Defining the bath tub a meters long $b$ meters wide and $c$ meters deep. Hot water flow into the bathtub in a constant speed $\left(\mathrm{c}_{\mathrm{f} 1}\right)$ from the left side. And the redundant water flow out from the right side in a constant speed $\left(\mathrm{c}_{\mathrm{f} 2}\right)$ after the bathtub is filled. So we simplify this model to the control volume model of steady flow. Using steady flow equation of open system in Engineering Thermodynamics to discuss this problem. Building a coordinate system as the Figure 1 and cut out a water lamina vertical to $y$-axis and analysis.

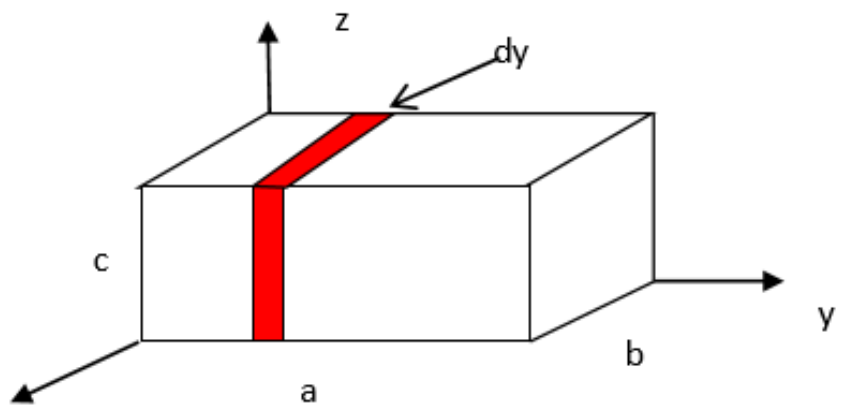

Figure 1 the simplified basic model

Step 1: Acquire the Temperature of Water Lamina of $y$-axis. According to the model simplified above. The water in the bathtub can be regard as working substance between high temperature source and low temperature source that distributed equally. So according to the “distance-time-temperature” model [1], the temperature of y-axis random position is 


$$
T=T_{1}-\frac{T_{1}-T_{2}}{a} y
$$

Step2: Calculation of Radiant Heat Dissipation. According to "The basic law of thermal radiation" .While the bathwater exchange heat with the atmosphere, we consider the exchange process as a dynamic process. So according to "Stefan-Boltzmann law" [2], the radiant heat flux of water lamina to the atmosphere in unit time is:

$$
\left\{\begin{array}{l}
d q_{1}=d A \cdot \varepsilon \sigma\left(T^{4}-T_{0}^{4}\right) \\
d A=b \cdot d y
\end{array}\right.
$$

Step 3: Calculation of Heat Transfer through the Bathtub Wall. According to the basic law of heat conduction[3], the bathtub water transfer the heat through bathtub wall steadily, this heat loss type can be seen as a "steady one-dimensional plate heat transfer". We can use "Fourier's law of heat conduction" [4] to gain the radiant heat dissipation from the bathtub wall per unit time:

$$
d q_{2}=-\lambda \cdot d S \cdot(d T / d x)
$$

Differential equation:

$$
\left\{\begin{array}{l}
d T / d x=-\left(d q_{2}\right) /(\lambda \cdot d S) d x \\
d S=(2 c+b) \cdot d y
\end{array}\right.
$$

Boundary condition:

$$
x=0, T=T ; x=b, T=T_{0}
$$

Integration:

$$
\int_{T}^{T_{0}} d T=-\int_{0}^{d} q_{2} /(\lambda \cdot d S) d x
$$

Radiant heat dissipation from the bathtub wall:

$$
d q_{2}=\frac{\lambda}{d} \cdot d S \cdot\left(T_{1}-T_{2}\right)
$$

Step 4: Calculation of Convection Heat Transfer between Water and Air. According to the definition of convection heat dissipation, the water in the bathtub is heat radiating from the upper surface to the air. We consider the converting process as a dynamic process. So by using " Newton's law of cooling" [5], the heat loss through convection per unit time is:

$$
d q_{3}=h_{c} A\left(T-T_{0}\right)
$$

Step 5: Similar to other steps. Integrating these three steps, we can acquire the sum of lamina heat dissipation:

$$
d q=d q_{1}+d q_{2}+d q_{3}
$$

That is:

$$
d q=\left[b \varepsilon\left(T(y)^{4}-T_{0}^{4}\right)+\left(\frac{(2 c+b) \cdot \lambda}{d}+b h_{c}\right)\left(T(y)-T_{0}\right)\right] d y
$$

The integration of the upper equation is the heat loss of bathwater to the atmosphere in unit time.

$$
Q=\frac{T_{1}-2 T_{0}+T_{2}}{2}\left[\frac{(b+2 c) \lambda}{d}+b h_{c}\right]+\frac{\varepsilon a b}{S\left(T_{2}-T_{1}\right)}\left(T_{2}^{5}-T_{1}^{5}\right)-\varepsilon a b T_{0}^{4}
$$

Step 6: Calculation of the Energy Difference Carried by the Inflow and Outflow Water per Unit Time.

According to the research of the open system in Engineering Thermodynamics, the energy carried by inflow water is

$$
E_{1}=\left(h_{1}+\frac{c_{f 1}^{2}}{2}+g z_{1}\right) q_{i n}
$$

The energy carried by outflow water is:

$$
E_{2}=\left(h_{2}+\frac{c_{f 2}^{2}}{2}+g z_{2}\right) q_{\text {out }}
$$


For the bathwater can be regarded as steady flow, the inflow and outflow water speed follows:

$$
c_{f 1}=c_{f 2}
$$

The inflow and outflow water yield in unit time has following relation:

$$
q=q_{\text {in }}=q_{\text {out }}
$$

The faucet and overflow are at the same height, so:

$$
z_{1}=z_{2}
$$

Thus, the energy difference between the inflow and outflow water per unit time :

$$
E_{1}-E_{2}=\left(h_{1}-h_{2}\right) \cdot q
$$

Final Step: Similar to other steps. According to the first law of thermodynamics [6], we get:

$$
Q=d E_{c v}+E_{1}-E_{2}+W_{i}
$$

Since the bathwater can be seen as constant temperature, we can realize the inner energy of the whole bathtub has no variation. That is:

$$
E_{c v}=0
$$

Since the inner water of bathtub has little friction, and there's no other work, so the internal work can be expressed:

$$
W_{i}=0
$$

So equation (1) can be simplified as:

$$
Q=\left(h_{1}-h_{2}\right) \cdot q
$$

According to the Engineering Thermodynamics, enthalpy $(h)$ is relative to the temperature $(T)$ and pressure $(P)$, and neglect the pressure change for it has minimal difference at different position.

Looking up the water enthalpy in different temperature, and using MATLAB to draw the curve graph whose $x$-axis is $T, y$-axis is $h$, which is shown as Figure 2.

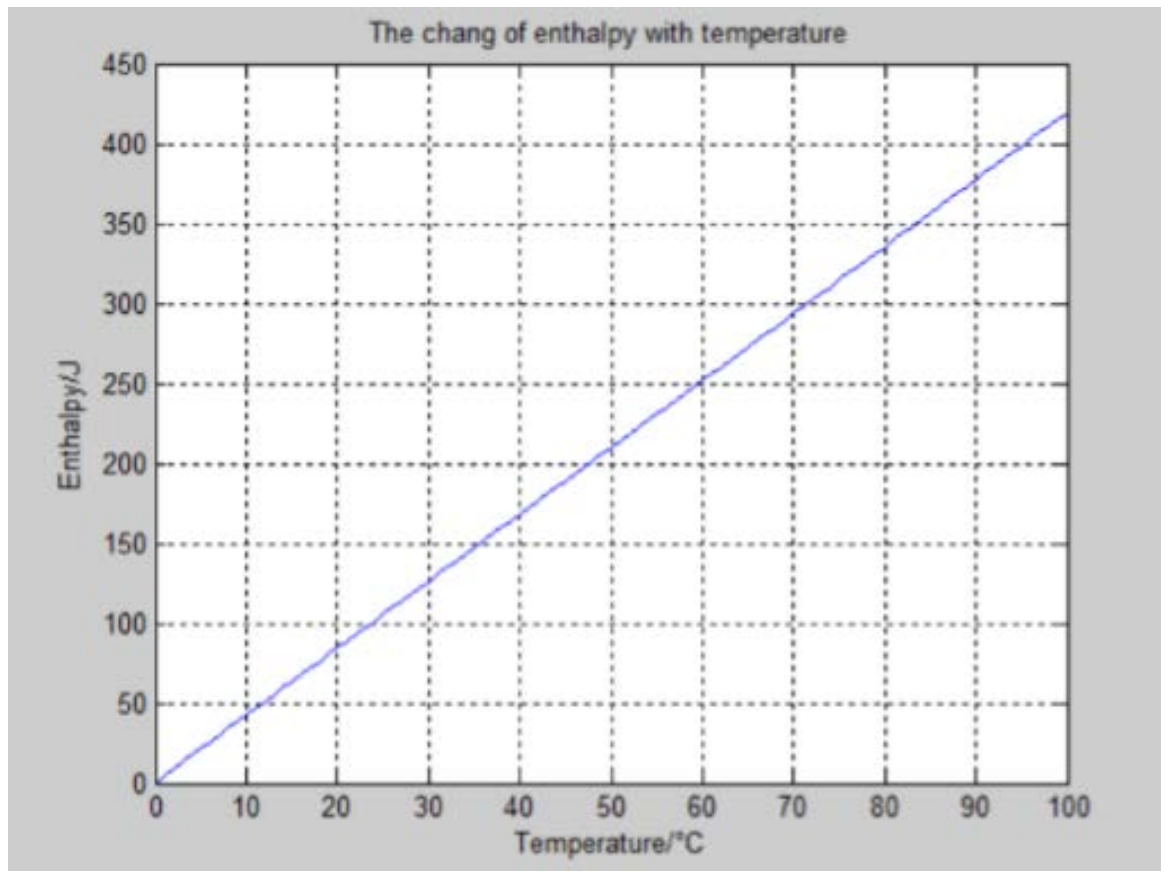

Figure 2 the change of enthalpy with temperature

As fitting the curve, we can acquire the connection between $\Delta h$ and $T$ ( $\Delta h$ : the enthalpy difference between the inflow and outflow water per unit time.)

$$
\Delta h=4.188\left(T_{1}-T_{2}\right)
$$

Integrated the above steps, we can acquire the mass of inflow water:

$$
Q=\frac{\frac{T_{1}-2 T_{0}+T_{2}}{2}\left[\frac{(b+2 c) \lambda}{d}+b h_{c}\right]+\frac{\varepsilon a b}{S\left(T_{2}-T_{1}\right)}\left(T_{2}^{5}-T_{1}^{5}\right)-\varepsilon a b T_{0}^{4}}{4.188\left(T_{1}-T_{2}\right)}
$$




\section{Conclusion}

The model is an optimization problem model. Assuming that the average temperature of bathtub water remains the same as the initial temperature, using software "Mathematic" to make bath shape parameters and the temperature of inflow and outflow water gain fixed values during a suitable range so as to get the optimal solution of the corresponding water mass flow. Increasing one of the decision variables, such as the inflow water temperature, the mass flux of water obtained by the model is reduced, which confirms to the objective facts, so the accuracy of the model was tested.

\section{References}

[1] distance-time-temperature: “A Research on distance-time-temperature curve model for heat condition in stored bulk grain.” http://www.docin.com/p-388755659.html?qq-pf-to=pcqq.c2c

[2] Stefan-Boltzmann law: Montvay I, Pietarinen E. The Stefan-Boltzmann law at high temperature for the gluon gas[J]. Physics Letters B, 1982, 110(2):148-154.

[3]Anderson D A, Tannehill J C, Pletcher R H. Computational fluid mechanics and heat transfer. Washington: Hemisphere. 2nd ed.1 997

[4] Fourier's law of heat conduction: Coleman B D, Mizel V J. Thermodynamics and departures from Fourier's law of heat conduction[J]. Archive for Rational Mechanics \& Analysis, 1963, 13(13): 245-261.

[5] Newton's law of cooling: O’Sullivan C T. Newton's law of cooling-A critical assessment[J]. American Journal of Physics, 1990, 58(10):956-960.

[6] Wei Wei, Jianqin Wu, Xiaodong Ma. On the first law of thermodynamics [J]. Journal of xinjiang normal university (natural science edition), 2011, (4) : 59-62. 